\title{
Ultrafast Spin Dynamics In Electrochemically Grown Heusler Alloy Films
}

\author{
Md Rejaul Karim ${ }^{1}$, Arundhati Adhikari ${ }^{2}$, Surya Narayan Panda ${ }^{2}$, \\ Purbasha Sharangi ${ }^{3}$, Soubhik Kayal ${ }^{4}$, Gouranga Manna ${ }^{5}$, P. S. Anil \\ Kumar $^{4}$, Subhankar Bedanta ${ }^{3}$, Anjan Barman ${ }^{2}$, and Indranil Sarkar ${ }^{1 *}$ \\ ${ }^{1}$ Institute of Nano Science and Technology, Sector 81, \\ Sahibzada Ajit Singh Nagar, Punjab 140306, India \\ ${ }^{2}$ Department of Condensed Matter Physics and Material Sciences, \\ S. N. Bose National Centre for Basic Sciences, \\ Block JD, Sector III, Salt Lake, Kolkata 700106, India \\ ${ }^{3}$ Laboratory for Nanomagnetism and Magnetic Materials (LNMM),
} School of Physical Sciences, National Institute of Science Education and Research, HBNI, Jatni 752050, Odisha, India

${ }^{4}$ Department of Physics, Indian Institute of Science, Bangalore 560012, India and ${ }^{5}$ Surface Physics Division, Saha Institute of Nuclear Physics, Kolkata 700064, India

\footnotetext{
* indranil.sarkar@inst.ac.in
} 
$\alpha$ shows an inverse relationship both with $\tau_{E}$ and $\tau_{M}$ [Fig. 9(c)-(d), main manuscript]. To help interpret this findings we look into the scattering mechanism where phonon mediated spin flip scattering drives the fast recovery process [1]. In 3TM, the remagnetization process involves the cooling of the hot-electron through energy transfer to the lattice approaching towards equilibration of both electron and lattice subsystems. This process is dominated by the phonon mediated (Elliot-Yafet) EY spin flip scattering that persists during the damping of precessional dynamics and connect both these phenomena. Alternatively, the magnon diffusion model to correlate remagnetization and Gilbert damping in ferromagnetic alloy systems has been proposed by Knut et al [2]. The ultrafast incoherent magnon generation due to interband transition of highly excited electrons and the electron-magnon interaction play crucial role in the ultrafast demagnetization and dominates over EY spin flip scattering during the remagnetization. They proposed that the decay of magnon is effectively determined by the Gilbert damping and leads to the remagnetization.

However, the detailed quantitative calculation correlating the distinct time scales of ultrafast magnetization dynamics has been carried out by inclusion of various models in some prior works. Koopmans et al. based on quantum mechanical treatment proposed that damping coefficient scales inversely to the demagnetization time assuming two different magnetic fields, i.e. exchange field and total homogeneous effective field (consisting of external field, anisotropy field and demagnetization field) dominate the effective magnetic field in femtosecond and nanosecond regime [3]. Later, by using ab-initio calculation Fahnle et al. have showed that $\alpha$ can be either proportional or inversely proportional to $\tau_{M}$ depending upon the dominating microscopic contribution to the magnetic damping [4]. The linear relation sustains when the damping is dominated by intraband mechanism driven conductivity-like contribution, whereas the interband mechanism driven resistivity-like contribution leads to an inverse relation between them. Recent report by Zhang et. al. shows that $\alpha$ is directly proportional to $\tau_{M}$ in $\mathrm{Co} / \mathrm{Ni}$ multilayer due to conductivity-like contribution in damping originated from breathing Fermi surface model [5]. They also suggest that an inverse relationship between $\alpha$ and $\tau_{M}$ can be attributed to the nonlocal spin current in metallic heterostructure system. More recently, Pan et al. has demonstrated an inverse relationship between $\alpha$ and $\tau_{E}$ for the half-metallic $\mathrm{Co}_{2} \mathrm{Fe}_{x} \mathrm{Mn}_{1-x} \mathrm{Si}$ Heusler alloy thin films that can be explained based magnon dominated remagnetization in Heusler alloy [6, 7]. Based on the above, we believe that the three stages of ultrafast magnetization dynamics are connected 
through different quasiparticles-scattering mechanism.

\section{REFERENCES}

[1] Roth, T; Schellekens, A. J.; Alebrand, S.; Schmitt, O.; Steil, D.; Koopmans, B.; Cinchetti, M.; Aeschlimann, M. Temperature Dependence of Laser-Induced Demagnetization in Ni: A Key for Identifying the Underlying Mechanism, Phys. Rev. X 2012, 2, 021006.

[2] Knut, R; Delczeg-Czirjak, E. K.; Jana, S; Shaw, J. M.; Nembach, H. T.; Kvashnin Y.; Stefaniuk, R.; Malik, R. S.; Grychtol, P; Zusin; D. et al., Inhomogeneous magnon scattering during ultrafast demagnetization, arXiv:1810.10994v1 [cond-mat.mtrl-sci].

[3] Koopmans, B; Ruigrok, J. J. M; Dalla Longa, F.; de Jonge W. J. M. Unifying Ultrafast Magnetization Dynamics Phys. Rev. Lett. 2005, 95, 267207.

[4] Fähnle, M. F.; Seib, J.; Illg, C. Relating Gilbert damping and ultrafast laser-induced demagnetization, Phys. Rev. B 2010, 82, 144405.

[5] Zhang, W.; He, W.;Zhang, X. -Q.; Cheng, Z. -H.; Teng, J.; Fahnle, M. Unifying ultrafast demagnetization and intrinsic Gilbert damping in $\mathrm{Co} / \mathrm{Ni}$ bilayers with electronic relaxation near the Fermi surface Phys. Rev. B 2017, 96, 220415 (R).

[6] Pan, S.; Seki, T.; Takanashi, K.; Barman, A.; Ultrafast demagnetization mechanism in halfmetallic Heusler alloy thin films controlled by the Fermi level Phys. Rev. B 2020101224412.

[7] Malik R S, Delczeg-Czirjak E K, Thonig D,Knut R,Vaskivskyi I,Gupta R, Jana S,Stefanuik R,Kvashnin Y O, Husain S, Kumar A, Svedlindh P, Söderström J, Eriksson O, and Karis O, Ultrafast magnetization dynamics in half-metallic $\mathrm{Co}_{2} \mathrm{FeAl}$ Heusler alloy, arXiv:2002.12255v1 [cond-mat.mtrl-sci]. 